SF 323

. W3

Copy 1

\title{
OFFICERS AND MEMBERS
}

OE THE

WASHINGTON JOCKEY CLUB. 



\section{OFEICERS \\ ов тив \\ WASHINGTON JOCKEY CLUB. \\ -0 :}

President,

CHARLES ASTOR BRISTED.

Vice Presidents,

Admiral D. D. Porter, Joseph H. Bradley, Esq. Dr. Chas. H. Nichols, Marshall Brown, Esq.

\section{Executive Committee.}

John W. Thompson, (Ch'm.,)

Richard Wallach,

Richard T. Merrick,

H. R. Rathbone,

A. S. Nicholson,

Samuel V. Niles,

Amos Webster,

Albert Ray,

O. E. Babcock,

I. N. Burritt,

John C. Cash,

William-Myers,

William B. Moses,
William H. Philip,

Thomas L. Hume,

S. S. Smoot,

William Marbury,

Richard Smith,

S. S. Carroll,

John A. Baker,

William G. Moore,

B. L. Jackson,

Jesse Brown,

James C. Kennedy,

Richard Harrington,

S. H. Kauffmann,

Wright Rives.

We the undersigned agree to contribute annually, the sums set opposite our names, respectively, to the funds of the Washington Jockey Club. 



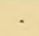

a. 
SF $32=$

W 3 
SF 32

W 3 
$S F 32^{\circ}$

W 3 



$$
\begin{aligned}
& S F 32 \\
& W / 3
\end{aligned}
$$


SF $32:$

W 3 



$$
\begin{aligned}
& S F 32 \\
& W 3
\end{aligned}
$$





$$
\begin{aligned}
& S F 32 \\
& W_{3}
\end{aligned}
$$





$$
\begin{aligned}
& S F 32 \\
& W / 3
\end{aligned}
$$



SF $32=$

W/ 3 

$S F 32^{2}$
$W$ 

SF $32=$

$\mathrm{W} / 3$ 



$$
\begin{aligned}
& \mathrm{SF} 32 \\
& \mathrm{~W} / 3
\end{aligned}
$$





$$
\begin{aligned}
& S F 32= \\
& W / 3
\end{aligned}
$$



SF 32

W 3 



$$
\begin{aligned}
& S F 32 \\
& W / 3
\end{aligned}
$$





$$
\begin{aligned}
& S F 32 \\
& W 3
\end{aligned}
$$





$$
\begin{aligned}
& \mathrm{SF} 32 \\
& \mathrm{~W} / 3
\end{aligned}
$$





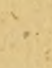

s 
LIBRARY OF CONGRESS

|||||||||||||||||||||||||||

00028295182 